\title{
Criterios \\ Nuevos compromisos para la gestión de la cartografía temática del Patrimonio Histórico de Andalucía
}

José Manuel Díaz Iglesias > Sandra Rodriguez de Guzmán

Dpto. de Técnicas Gráficas. Centro de Documentación del IAPH

\section{Resumen}

La tarea de gestión de la cartografia digital del Patrimonio Histórico de Andalucia supone un compromiso con un servicio público de la administración regional. Esta labor se va a ver afectada por la futura ordenación de la actividad cartográfica de Andalucía, lo cual va a exigir la adopción de una serie de decisiones por parte de la Consejería de Cultura para asumir las obligaciones que se deducen de su participación en el nuevo modelo propuesto. En estas líneas se pretende avanzar, desde la experiencia del IAPH, una reflexión sobre la implicación de la Consejería de Cultura en dicho modelo.

Palabras clave

Patrimonio Histórico

Información espacial

Cartografia

Metadatos

Calidad

Servicio Público

Infraestructura de Datos Espaciales
La cartografía digital del Patrimonio

Histórico de Andalucía entre los servicios públicos de la Administración

En el año 1996 el Centro de Documentación del Instituto Andaluz del Patrimonio Histórico (IAPH) inició las primeras tareas para implementar un Sistema de Información Geográfica (SIG) asociado al Sistema de Información del Patrimonio Histórico de Andalucía (SIPHA). El objetivo principal era dar respuesta a la necesidad de contar con las herramientas y los datos necesarios que posibilitasen la gestión y la investigación del Patrimonio Histórico de forma integrada como un elemento más vertebrador del territorio.

Desde un primer momento, las líneas de actuación se centraron fundamentalmente en el levantamiento de la información espacial, el desarrollo de las herramientas informáticas que garantizasen tanto la administración como la consulta de los datos y, por último, la formación de los técnicos.

Pese a la importancia de las tres actuaciones, sin duda los mayores esfuerzos se aplicaron a la creación de la información espacial. Si bien inicialmente se trabajó de forma sectorial con el Patrimonio Arqueológico, paulatinamente se fueron incorporando otras tipologías patrimoniales. De este modo, entre 1996 y 1997, se realizó una primera cobertura digital del Patrimonio Arqueológico de Andalucía en colaboración con el Departamento de Prehistoria y Arqueología de la Universidad de Sevilla (Fernández, 2002). Esta experiencia se amplió posteriormente a los Conjuntos Históricos y el Patrimonio Arquitectónico (GallianiSánchez, 1998) y desde 1999 el IAPH se encargó directamente de esta labor para la totalidad del Patrimonio Inmueble. Fruto de esta tarea, en la actualidad, la cartografía digital del Patrimonio Histórico cuenta con un número aproximado de 15.000 registros espaciales.

Para la administración de este importante volumen de información se hacia imprescindible la adecuación de una serie de parámetros y controles que asegurasen la integridad y calidad de la misma. Para ello se desarrollaron herramientas que permitiesen de forma automática la actualización de las modificaciones de la información espacial y alfanumérica así como la localización de errores en la información geográfica. De nuevo, la primera herramienta se aplicó al trabajo con la cartografía digital del patrimonio arqueológico (Fernández-Blasco-Fernández, 2000) y con posterioridad se hizo extensiva a todo el Patrimonio Histórico.

De forma paralela el IAPH, como representante de la Consejería de Cultura, pasó a formar parte de la Comisión de Cartografía de Andalucia, colaborando con el Instituto de Cartografía de Andalucía (ICA) y otras Consejerías mediante la aportación de la car- 


\section{Criterios \\ Nuevos compromisos para la gestión de la cartografía temática del Patrimonio Histórico de Andalucía \\ J. M. Díaz Iglesias S. Rodríguez de Guzmán}

tografía generada y la adopción de los diferentes criterios técnicos y metodológicos sugeridos.

A medida que se desarrollaba la cartografía temática del Patrimonio Histórico, ésta fue adquiriendo una doble utilidad como instrumento para el conocimiento y la gestión del territorio. Por un lado, en el contexto de la labor de la Consejería de Cultura y unida a las herramientas que hacen posible su tratamiento, se convertía en un elemento de apoyo para el desarrollo de una adecuada política de gestión. Por otro lado, en el marco de los proyectos con implicación territorial desarrollados por otras consejerías o empresas privadas, resultaba fundamental para proporcionar la correcta localización y delimitación de los diferentes bienes con el objetivo de garantizar tanto la integridad de los mismos como el cumplimiento de las determinaciones que se desprendían de su situación legal.

Atendiendo a esto último, el Centro de Documentación del IAPH comenzó a proporcionar un nuevo servicio mediante el cual se facilitaba cartografía digital del Patrimonio Histórico de las zonas afectadas por actuaciones que requiriesen el conocimiento de la localización de los Bienes Inmuebles. Este servicio acaba de quedar fijado recientemente en la publicación de la Carta de Servicios del IAPH (Blanco-Ortega, 2005) mediante la Resolución de 17 de enero de 2005 de la Dirección General de Bienes Culturales ${ }^{1}$. La finalidad de este documento, según se recoge en el artículo 4 del Decreto ${ }^{2}$ que regula los mismos, se puede resumir en tres aspectos: informar a los usuarios de los servicios que se gestionan, de las condiciones en la que se prestan y de los compromisos de calidad que se asumen junto a ellos.

Este tipo de documentos se inserta dentro de los retos asumidos en el marco de la Sociedad de la Información por la Administración regional y tienen en común el compromiso de facilitar las herramientas y tecnologías necesarias para obtener información integrable de forma sencilla y con suficientes garantías de calidad. Para ello, la Junta de Andalucía ha puesto en marcha en los últimos años diversas iniciativas con la finalidad de mejorar e incrementar los servicios públicos, al tiempo que avanzar en la calidad de los mismos (Sendra Arce, P.J., 2004).

En este sentido, pero en el ámbito de la producción y difusión cartográfica, la futura publicación del Decreto de Ordenación de la Cartografía Andaluza (actualmente en fase de redacción) por la Consejería de Obras Públicas y Transportes va a incidir en la misma idea al entender la información cartográfica como un instrumento útil para la sociedad y por tanto como un servicio público, siendo tarea de la administración regional facilitar el acceso a dicha información, proporcionando los medios técnicos y tecnológicos necesarios así como definiendo y asumiendo los compromisos de calidad necesarios.
En estas líneas se pretende esbozar la implicación de la Consejería de Cultura en el futuro ordenamiento y plantear las obligaciones que de la misma se deducen. Para ello se hará especial hincapié en el proyecto de Directiva INSPIRE, como iniciativa europea que comparte los objetivos de la administración autonómica ya citados. Aunque el Decreto se encuentra aún en fase de borrador, y por tanto es previsible que sufra modificaciones antes de su publicación, esto no anula las conclusiones que de forma genérica pueden extraerse del mismo. Con ellas se pretende propiciar una reflexión de cara a las estrategias y actuaciones que deberian asumirse por parte de la Consejería de Cultura.

\section{La Consejería de Cultura en la futura ordenación de la cartografía andaluza}

La aprobación del futuro Decreto de Ordenación de la Cartografía Andaluza se enmarca dentro de la necesidad de regular y adecuar la producción y difusión de la cartografía regional al surgimiento de una serie de nuevos requisitos:

$>$ La necesidad de establecer mecanismos que se adecuen al incremento de la demanda de información georreferenciada detectado en el marco de la Sociedad de la Información y el Conocimiento.

$>$ La importancia de estructurar un modelo coherente que se adapte a las necesidades de cooperación entre las diferentes consejerías, instituciones y corporaciones locales de la comunidad autónoma.

> Regular la función de la cartografía andaluza para que se amolde tanto a las necesidades derivadas de la conformación de un sistema cartográfico nacional como a las iniciativas tomadas por la Unión Europea en el marco del proyecto de la Directiva INSPIRE.

Para ello se toma como punto de partida la experiencia recogida a lo largo de una trayectoria que va desde la creación del ICA hasta la actualidad. A lo largo de este tiempo ha venido funcionando un modelo coordinado y programado por este organismo donde un porcentaje de la producción cartográfica ha estado descentralizado, siendo las distintas consejerías y entidades autonómicas con responsabilidad sobre esta materia las que se han ocupado de esta labor.

La Consejería de Cultura ha venido desarrollando dentro de ese modelo una actividad cartográfica centrada fundamentalmente en la planificación, creación y administración de la cartografía temática de Patrimonio Histórico. Conviene precisar que cuando se menciona la labor de la Consejería de Cultura en la futura ordenación se está haciendo referencia, hasta el día de hoy, a la labor en materia cartográfica de una de sus cuatro Direcciones Generales, concretamente la de Bienes Culturales. 
La regulación de la actividad cartográfica en Andalucia supondrá una continuidad del modelo actual en sus líneas básicas aportando como novedad la ordenación de algunos aspectos organizativos con el fin de adecuar la producción cartográfica a las necesidades ya mencionadas. El conjunto de estas novedades, en lo que se refiere a sus implicaciones en la actividad cartográfica de nuestra Consejería, se puede resumir de forma genérica en cuatro apartados:

$>$ Consideración oficial de su producción cartográfica

$>$ Necesidad de definir una estructura jerárquica de organización de las diferentes unidades administrativas implicadas en la producción cartográfica

> Representación de la Consejería en el Consejo y la Comisión de Cartografía

> Necesidad de determinar su participación en la Infraestructura de Datos Espaciales de Andalucia

\section{La Cartografía Oficial}

El primer punto hace referencia a la consideración de Cartografía Oficial que se otorga a la producción cartográfica de esta Consejería. Para ello se exigirá el cumplimiento de tres requisitos básicos: estar desarrollada bajo una norma técnica que esté sometida a un control de calidad validado por el ICA, ser accesible públicamente y ser actualizada periódicamente. Estas tres condiciones ya se cumplen actualmente y sólo es necesario tenerlas en consideración de cara a mantenerlas y mejorarlas en futuras actuaciones.

En relación con el primer punto, es necesario señalar que la Dirección General de Bienes Culturales se encuentra en estos momentos inmersa en diversas tareas encaminadas al desarrollo del futuro Sistema de Información de los Bienes Culturales de AndaIucía MOSAICO (Consejería de Cultura. Junta de Andalucia, 2005). Como parte de las mismas se han creado una serie de grupos de trabajo, de los cuales uno de ellos está encargado del análisis de las necesidades y requisitos para la implantación del futuro Sistema de Información Geográfica. Como parte de las labores de este grupo se ha asumido la realización de un documento de Normas Técnicas para el levantamiento y producción de la cartografía temática patrimonial (AA.WV, 2004).

Con respecto al requisito de ser accesible públicamente, ya se ha mencionado su inclusión en la Carta de Servicios del IAPH. Pero además de esto, la consideración de cartografía oficial implicará que ésta forme parte del Registro andaluz de Cartografía, el cual se creará y será de acceso público.

En cuanto a la tercera condición, se trata de una característica inherente a la producción cartográfica y está garantizada, en la actualidad, con la alimentación de la Base de Datos del SIPHA y con el empleo de las diferentes herramientas que facilitan esta tarea de forma automática.

¿Qué implica la consideración oficial de la producción cartográfica? Básicamente que la cartografía generada por nuestra Consejería es de uso obligado en cualquier procedimiento administrativo que requiera la representación geográfica precisa del Patrimonio Histórico, tanto por la Administración Pública como por los particulares en su relación con ésta.

Esto supone un respaldo legal que debe redundar doblemente en beneficio de la gestión y protección del Patrimonio Histórico. En primer lugar, porque define el área de competencias y responsabilidades en materia cartográfica de esta Consejería. Por otra parte, porque obliga a otras consejerías con competencias en la gestión del territorio a utilizar y, por tanto, a conocer la cartografía temática de Patrimonio Histórico.

\section{Representación de la Consejería en el Consejo y la Comisión de Cartografía}

El futuro documento normativo establecerá que las labores de coordinación de la actividad cartográfica, de participación en la misma, y de cooperación entre las diferentes consejerías y organismos, se llevarán a cabo en el Consejo de Cartografía (de nueva creación) y la Comisión de Cartografía.

En ambos órganos la Consejería de Cultura contará con un representante. Actualmente tanto el IAPH como la Dirección General de Bienes Culturales forman parte de la Comisión de Cartografía de Andalucía.

La experiencia de esta participación ha demostrado la importancia de este último órgano como coordinador de la producción cartográfica y foro de conocimiento tanto de las diversas iniciativas emprendidas en materia cartográfica por los diversos agentes implicados en la misma como de las disposiciones normativas relacionadas.

Esta misma experiencia aconseja la designación de representantes estrechamente relacionados con las labores de coordinación de la actividad cartográfica con el fin de evitar la desconexión entre ambas tareas.

Definición de una estructura jerárquica y coordinada con diferentes Unidades de producción Cartográfica

La producción cartográfica en nuestra comunidad hasta la actualidad se ha caracterizado por la existencia de un modelo jerárquico descentralizado donde el ICA ha actuado como organismo cabecera y coordinador de la producción cartográfica, que en parte ha recaido sobre las distintas Consejerias que asumian 


\section{Criterios \\ Nuevos compromisos \\ para la gestión de la \\ cartografía temática del \\ Patrimonio Histórico de \\ Andalucía \\ J. M. Díaz Iglesias \\ S. Rodríguez de Guzmán}

esta labor en función de las necesidades que se desprendian del desarrollo de sus competencias.

Este modelo se mantiene en la nueva propuesta, pero como novedad se plantea la necesidad de crear en cada consejería una 'Unidad Cartográfica', siendo obligatoria su existencia en aquéllas que cuenten con un representante en el Consejo de Cartografía. Bajo la coordinación de ésta podrán crearse tantas unidades como se estimen oportunas.

En el caso de la Consejería de Cultura, las funciones de la Unidad Cartográfica serian las de coordinar las actuaciones en esta materia, elaborar propuestas de nuevas actividades para incluirlas en el Plan Cartográfico de Andalucía y participar en la coordinación global de la actividad cartográfica regional.

Hasta estos momentos, dentro de la estructura general de la Consejería de Cultura, sólo la Dirección General de Bienes Culturales desarrolla una labor que conlleva necesariamente un contacto a diferentes niveles con la complejidad de la gestión territorial. Esto implica la necesidad de trabajar con un Sistema de Información Geográfica. Como se ha señalado en la introducción, el IAPH ha trabajado en el desarrollo e implantación de un SIG, habiendo puesto en marcha como parte del mismo los protocolos de trabajo, la formación especializada de los técnicos implicados y las infraestructuras necesarias, entre ellas, el conjunto de datos espaciales que constituyen la cartografía digital del Patrimonio Histórico.

Por todo ello, el IAPH ha venido funcionando, a los efectos que se determinan en el futuro documento normativo, como Unidad Cartográfica de la Consejería de Cultura.

Sin embargo, en el marco general del nuevo modelo de ordenación cartográfica y de los nuevos desarrollos emprendidos por la Consejeria de Cultura, se hace necesario redefinir esta organización, determinando las competencias y funciones de los diferentes departamentos con atribuciones en el proceso de producción cartográfica.

\section{La participación en la Infraestructura de Datos Espaciales de Andalucía}

La creación de la Infraestructura de Datos Espaciales de Andalucía forma parte de las actuaciones iniciadas en el ámbito autonómico por el ICA con el objetivo de facilitar la difusión y el acceso a la información geográfica de esta comunidad. Su creación y puesta en marcha se inserta dentro de las acciones emprendidas a nivel nacional y europeo en este mismo sentido en relación con los objetivos de la Directiva INSPIRE (INnfraestructure for SPatial InfoRmation in Europe).

Este proyecto es una iniciativa de la Dirección General de Medio Ambiente de la Unión Europea, la Agencia Europea Eurostat y el
Centro de Investigaciones común JRC con el objetivo de crear una Infraestructura de Datos Espaciales (IDE) europea que proporcione a los usuarios servicios de identificación, localización y acceso a la información espacial generada en diferentes ámbitos: local, regional, nacional y supranacional (Mas-Vallejo, 2003).

Los Principios básicos por los que se rige el Plan de Acción de INSPIRE son los siguientes:

> Los datos espaciales son recogidos una vez y mantenidos en el nivel donde se logra máxima efectividad

$>$ Debe ser posible combinar información geográfica con total continuidad para toda Europa desde fuentes diversas, y compartirla entre usuarios y aplicaciones

> Debe ser posible que la información recogida en un nivel sea compartida por otros niveles

> La información geográfica debe ser abundante y disponible bajo condiciones que no inhiban su uso extensivo

$>$ Debe ser fácil localizar la información geográfica disponible, y en qué condiciones puede conseguirse y usarse

$>$ Los datos espaciales deben ser fáciles de entender e interpretar y seleccionables amigablemente

El proceso para la puesta en marcha de la IDE europea comenzó con la designación de un grupo de expertos. Éstos a su vez establecieron cinco grupos de trabajo con la finalidad de preparar los documentos básicos que constituirían el pilar normativo para la puesta en marcha de la IDE Europea y la base doctrinal para desarrollar las IDE nacionales, regionales y locales. Estos documentos fueron aprobados en octubre del año 2002 (Mas-Vallejo, 2003).

En España, el Consejo Superior Geográfico asumió en abril de 2001 el desarrollo de la IDE de España y se lo encargó a su Comisión de Geomática, la cual comenzó los trabajos en noviembre de 2002. Para ello creó un grupo de trabajo que a su vez se organizó en cuatro subgrupos: Datos de Referencia, Metadatos, Arquitectura y Normas, Política de datos, precios y licencias (Rodriguez-Luján-Mezcua, 2003).

En Andalucía, de forma paralela al desarrollo de los trabajos a nivel nacional, se pusieron en marcha desde el ICA diversas iniciativas en este sentido, siendo presentada oficialmente en febrero de 2003 en el marco de una Ponencia Técnica de la Comisión de Cartografía de Andalucía (Garrido, M.T., 2003).

Esta trayectoria desarrollada a lo largo de los últimos cuatro años ha tenido como eje central, tal y como se ha expuesto, la crea- 
ción y puesta en marcha de las Infraestructuras de Datos Espaciales bajo el auspicio y la coordinación del Instituto Geográfico Nacional y de sus equivalentes regionales.

Si se tienen en consideración las características generales de las diferentes iniciativas realizadas bajo el objetivo común que comparten, una definición de las IDE debería tener en cuenta los aspectos organizativos y tecnológicos, además de la voluntad institucional, como elementos claves que posibilitan la normalización de la producción cartográfica con el fin de facilitar el acceso a la información espacial (Capdevila, J., 2004). Todo ello sin olvidar el núcleo fundamental de la información espacial y de los descriptores de la misma, los metadatos.

En el ámbito de la administración andaluza, la voluntad institucional se ha puesto de manifiesto con el impulso dado desde el ICA a la creación de la IDE de Andalucia y, de igual modo, con la inscripción de la misma en el Plan de Iniciativas Estratégicas para la Sociedad de la Información (Garrido, M.T., 2003).

En cuanto a los aspectos organizativos y tecnológicos, todo apunta hacia la Secretaría General de Telecomunicaciones y Sociedad de la Información de la recién creada Consejería de Innovación, Ciencia y Empresa como unidad institucional encargada de facilitar los mecanismos informáticos que permitan consultar y acceder a los datos espaciales administrados por la misma.

En lo referente a los Datos Espaciales y a los metadatos a ellos asociados, es aquí donde se centra la labor de la Consejería de Cultura, siendo necesario definir su participación y asumir las obligaciones de la misma.

La organización de los Datos Espaciales para la IDE de Andalucía se rige por las determinaciones que en este apartado ha fijado el grupo de trabajo específico de INSPIRE. De este modo se establece la existencia de dos bloques de información organizados en Datos Espaciales de Referencia y Datos Temáticos.

Son Datos de Referencia (Mas-Vallejo, 2003) "el conjunto de ficheros que todos los relacionados con el uso de información geográfica utilizan para referenciar sus datos" y "los datos que proporcionan un enlace común entre aplicaciones y por tanto un mecanismo para compartir conocimientos e información". En la IDE de Andalucía tienen la consideración de cartografía básica.

Los Datos Temáticos son el conjunto de información sectorial que se ha designado para su inclusión en función de las necesidades del sector medioambiental. En la IDE de Andalucía tienen la consideración de cartografía temática.

En principio, el Patrimonio Histórico no figura explícitamente en ninguno de los dos apartados aunque sí se ha tenido en cuenta esta posibilidad y no se descarta su inclusión a medio plazo. En uno de los últimos documentos ${ }^{4}$ del Grupo de trabajo INSPIRE, en el que se replantean los objetivos y el alcance de las previsiones originales, se propone una reducción del conjunto de datos espaciales propuestos inicialmente. Para ello establecen en tres anexos el listado definitivo de datos espaciales que se corresponden con los Datos de Referencia, los Datos Temáticos y un conjunto de datos temáticos que inicialmente quedan fuera, pero que han de ser considerados de cara a su posible inclusión en un futuro, entre los que figura el Patrimonio Cultural.

Los argumentos para la exclusión de este último conjunto de datos se centran en los resultados de los diferentes análisis realizados con el fin de determinar las necesidades más comunes de los usuarios. Sin embargo, también se establece que en función de las necesidades a otros niveles se podrán ampliar tanto los Datos de Referencia como los temáticos.

En este sentido debería proponerse en el marco de la IDE de Andalucía la inclusión de la cartografía de Patrimonio Histórico. Al menos inicialmente, los datos espaciales referidos a los bienes incluidos en el Catálogo General del Patrimonio Histórico podrían tener cabida de forma específica entre los Datos de Referencia, en el subapartado de 'Espacios Protegidos' bajo 'Limites Administrativos'. Las determinaciones de INSPIRE ${ }^{5}$ describen este conjunto de datos del siguiente modo: Protected Sites: Area designated or regulated and managed to archieve specific conservation objetives. Dado el marcado carácter medioambiental de INSPIRE, podría proponerse una diferenciación en este subapartado entre Espacios protegidos naturales y culturales.

Para finalizar, hay un último aspecto que ha de ser asumido obligatoriamente como parte de las labores de producción y administración de nuestra cartografía con el objetivo de conocer tanto las características de la información espacial a la que van unida como los parámetros de calidad de la misma. Se trata de la adopción y cumplimentación de los metadatos, lo cual será obligatorio en el marco general del nuevo modelo de ordenación de la cartografía andaluza.

Los metadatos, como descriptores de la calidad de los datos, van más allá de la descripción del grado de fidelidad alcanzado en la modelización de la realidad, permitiendo además contextualizar los datos espaciales de cara a favorecer el intercambio de los mismos y en definitiva una correcta difusión.

Para asegurar un lenguaje común que favorezca la comunicación entre los diferentes productores y, entre éstos y los posibles usuarios, se han establecido diversas propuestas estándares de metadatos de información geográfica. Sin entrar en las mismas, 


\section{Criterios \\ Nuevos compromisos para la gestión de la cartografía temática del Patrimonio Histórico de Andalucía \\ J. M. Díaz Iglesias S. Rodríguez de Guzmán}

sólo se pretende señalar que aún no se ha adoptado un núcleo común aunque todo parece indicar que, dada la amplitud y complejidad del estándar ISO TC/211 19115 que INSPIRE propone, se recomendará una versión más reducida en el ámbito de la producción cartográfica nacional, el denominado Núcleo Español de Metadatos (NEM). Esta propuesta está formada por los descriptores extraídos del núcleo básico ISO 19115 y del Dublin Core más una serie adicional añadida por el subgrupo de trabajo de la IDE de España hasta conformar un total de 29 descriptores (Capdevila, J., 2004).

En todo caso, y para finalizar, el ICA será el organismo encargado de trasladar las determinaciones que se acuerden sobre este aspecto a los distintos agentes implicados en la producción cartográfica.

\section{Conclusiones}

Las diferentes iniciativas que la Junta de Andalucia está llevando a cabo en el marco de la Sociedad de la Información y el Conocimiento, con el objetivo de adecuar los servicios que proporciona la administración regional a la creciente demanda de información, tienen como punto en común facilitar la difusión de los mismos.

La futura ordenación de la actividad cartográfica en nuestra comunidad está dentro de estas líneas de acción. La Consejería de Cultura, como parte de la administración pública, está obligada a trabajar en este sentido, adecuando la producción de su cartografía temática a las necesidades señaladas. El conocimiento del Patrimonio Histórico a través de su representación cartográfica sólo puede repercutir en beneficio del desarrollo de sus competencias.

En este sentido se han presentado las principales implicaciones que para esta Consejería van a suponer la entrada en vigor de un nuevo marco de actuaciones. El esfuerzo realizado en los últimos años en materia cartográfica debe continuar con la adopción de los nuevos requisitos.

Para ello, los trabajos emprendidos desde la Consejería de Cultura para el desarrollo del citado proyecto MOSAICO van a suponer el marco adecuado para el planteamiento de las líneas de acción oportunas.

\section{Notas}

${ }^{1}$ Publicada en el Boletín Oficial de la Junta de Andalucía. № 33,16 de febrero de 2005, p. 16 y ss

2 Decreto 317/2003, de 18 de noviembre. Publicado en el Boletín Oficial de la Junta de Andalucía. $N^{\circ} 225,21$ de noviembre de 2003, p. 24513 y ss.
${ }^{3}$ Se cita el resumen que estos autores hacen del documento original que puede consultarse en: http://inspire.jrc.it/reports/position_papers/ inspire_rdm_pp_v4_3_en.pdf

${ }^{4}$ Se puede consultar en: http://inspire.jrc.it/reports/inspire_scoping24mar04.pdt

${ }^{5}$ Véase nota 4

\section{Bibliografía}

AA.VV. (2004) "Informe sobre la situación actual de MOSAICO, Subsistema de Información de los Bienes Culturales de Andalucia" presentado en I Jornadas Técnicas sobre el Plan de Sistemas de Información de la Administración Cultural. Antequera, Málaga

BLANCO, Á.; ORTEGA, I. (2005) La Carta de Servicios del Instituto Andaluz del Patrimonio Histórico. PH Boletín del IAPH, nº 53, 2005, p.78-73

CAPDEVILA I SUBIRANA, J. (2004) Infraestructura de datos espaciales (IDE). Definición y desarrollo actual en España. Geo Critica / Scripta Nova. Revista electrónica de geografía y ciencias sociales. Barcelona: Universidad de Barcelona. Vol. VIII, núm. 170-61. <http://www.ub.es/geocrit/sn/sn-170-61.htm>

CONSEJERÍA DE CULTURA. JUNTA DE ANDALUCíA (2005) La Consejería de Cultura fija las bases para un cambio cultural en la gestión administrativa. PH Boletín del IAPH, $n^{0}$ 52, 2005, p. 6-7

FERNÁNDEZ CACHO, S. (2002) La introducción de los SIG'S en la gestión de la Información arqueológica : GeoARQUEOS en ARQUEOS. Sistema de Información del Patrimonio Arqueológico de Andalucía. Cuadernos Técnicos, $n^{\circ}$ 6. Granada: Consejería de Cultura de la Junta de Andalucía

FERNÁNDEZ CACHO, S.; BLASCO ARANDA, E.; FERNÁNDEZ VICTORIO, R. (2000) GeoARQUEOS: A System for the Creation, Updating and Validation of the digital cartography of the Andalusian Archaeological Heritage. Archaeologia e Calcolatori, $n^{0} 11,1995$, p. 359-373 Universitá di Siena

GALLIANI, D.; SÁNCHEZ DÍAZ, F. J. (1998) Desarrollo de la base de datos geográfica de conjuntos históricos y patrimonio arquitectónico. PH Boletín del IAPH, $n^{\circ} 22,1998$, p. $102-105$

GARRIDO BORREGO, M. T. (2003) La IDE Andalucia, un compromiso de todos. MAPPING: Revista de Cartografía, Sistemas de Información Geográfica, Teledetección y Medio Ambiente, $n^{\circ} 86$

MAS MAYORAL, S.; VALLEJO BOMBÍN, R. (2003) El proyecto de directiva europea INSPIRE. MAPPING: Revista de Cartografía, Sistemas de Información Geográfica, Teledetección y Medio Ambiente, $n^{\circ} 86$

RODRígueZ, A.; LUJÁN, A.; MEZCUA, J. (2003) IDEE: la Infraestructura de datos española. MAPPING: Revista de Cartografia, Sistemas de Información Geográfica, Teledetección y Medio Ambiente, $n^{\circ} 86$

SENDRA ARCE, P. J. (2004) La Administración de la Junta de Andalucía en busca de la excelencia. PH Boletín del IAPH, $n^{\circ} 51,2004$, p. 88-93 